\title{
A new model for the infrared emission of IRAS F10214+4724
}

\author{
Andreas Efstathiou ${ }^{1}$, Natalie Christopher ${ }^{2}$, Aprajita Verma ${ }^{2}$, and \\ Ralf Siebenmorgen ${ }^{3}$ \\ ${ }^{1}$ School of Sciences, European University Cyprus, Engomi, 1516 Nicosia, Cyprus \\ ${ }^{2}$ Oxford Astrophysics, Denys Wilkinson Building, University of Oxford, Keble Rd, Oxford \\ OX1 3RH, United Kingdom \\ ${ }^{3}$ European Southern Observatory, Karl-Schwarzschildstr. 2, \\ 85748 Garching b. Munchen, Germany \\ email: a.efstathiou@euc.ac.cy
}

\begin{abstract}
We present a new model for the infrared emission of the high redshift hyperluminous infrared galaxy IRAS F10214 +4724 which takes into account recent photometric data from Spitzer and Herschel that sample the peak of its spectral energy distribution. We first demonstrate that the combination of the AGN tapered disc and starburst models of Efstathiou and coworkers, while able to give an excellent fit to the average spectrum of type 2 AGN measured by Spitzer, fails to match the spectral energy distribution of IRAS F10214+4724. This is mainly due to the fact that the $\nu S_{\nu}$ distribution of the galaxy falls very steeply with increasing frequency (a characteristic of heavy absorption by dust) but shows a silicate feature in emission. We propose a model that assumes two components of emission: clouds that are associated with the narrow-line region and a highly obscured starburst. The emission from the clouds must suffer significantly stronger gravitational lensing compared to the emission from the torus to explain the observed spectral energy distribution.
\end{abstract}

Keywords. galaxies: evolution, galaxies: formation, galaxies: high-redshift, infrared: galaxies, cosmology: observations

\section{Introduction}

The title of the discovery paper of IRAS F10214+4724 at a redshift of 2.285 (RowanRobinson et al. 1991) was 'An IRAS galaxy with huge luminosity - hidden quasar or protogalaxy?'. It soon became clear that the reason for the enormous luminosity was that the galaxy was gravitationally lensed by a factor of 10-100 (e.g. Broadhurst \& Lehar 1995). Until recently, however,it was not clear whether the dominant energy source was an active galactic nucleus (AGN) or a starburst.

Models for the infrared spectral energy distribution (SED) of IRAS F10214+4724 in the mid-90s remained inconclusive (e.g. Rowan-Robinson et al. 1993) mainly due to the limited wavelength coverage. Mid-infrared spectrophotometry has been shown for some time now to be able to differentiate between a starburst and AGN origin of the infrared emission (Roche et al. 1991 and references therein, Genzel et al. 1998). The mid-infrared spectrum of IRAS F10214+4724 obtained by Teplitz et al. (2006) using the IRS onboard Spitzer showed a puzzling emission feature at rest frame $10 \mu \mathrm{m}$ instead of the expected absorption feature given the type 2 nature of the AGN suggested by optical observations. Efstathiou (2006) proposed that the SED of IRAS F10214+4724 could be understood in terms of three components of emission: clouds that are associated with the narrow-line region and with a covering factor of 17\%, an edge-on torus and a highly 
obscured starburst. It was also noted by Efstathiou (2006), however, that because the emission from the torus and the narrow-line region clouds arises from diferent regions their magnification may be different.

In this paper we develop a new model for the infrared emission of IRAS F10214+4724 which takes into account new photometric data from Spitzer and Herschel that cover the wavelength range $70-170 \mu m$ and therefore complete its SED.

\section{Radiative transfer models}

\subsection{Starburst models}

Efstathiou, Rowan-Robinson \& Siebenmorgen (2000) developed a starburst model that has three main features: The first feature of the model is that it incorporates the stellar population synthesis model of Bruzual \& Charlot that gives the spectrum of the stars as a function of their age. Efstathiou, Rowan-Robinson \& Siebenmorgen (2000) used the table that assumes a Salpeter IMF and stellar masses in the range 0.1-125 $M_{\odot}$. The second important feature of the model is that it involves detailed radiative transfer that takes into account multiple scattering from grains and incorporates a dust model that includes small transiently heated grains and PAH molecules as well as large classical grains. For the calculation of the emission of the small grains and PAHs the method of Siebenmorgen \& Krügel (1992) was employed. The third feature of the model is that it incorporates a simple model for the evolution of the giant molecular clouds that constitute the starburst once a stellar cluster forms instantaneously at the center of the cloud. The most important characteristic of this evolutionary scheme is that by about 10 Myrs after star formation, the expansion of the HII region leads to the formation of a cold narrow shell of gas and dust. This naturally explains why the mid-infrared spectra of starburst galaxies are dominated by the PAH emission and not by the emission of hot dust. The sequence of molecular clouds at different ages can be convolved with a star formation history to give the spectrum of a starburst at different ages. Farrah et al. (2003) showed that the starburst models of Efstathiou, Rowan-Robinson \& Siebenmorgen (2000) in combination with the tapered discs of Efstathiou \& Rowan-Robinson (1995), which are described below, provided good fits to the SEDs of 41 ultraluminous infrared galaxies. One interesting prediction of the Efstathiou, Rowan-Robinson \& Siebenmorgen (2000) model is that young starbursts show strong near-infrared continuum, small $6.2 \mu \mathrm{m}$ equivalent widths and deep silicate absorption features and can therefore explain the galaxies in class 3A of Spoon et al. (2007). The starburst model has recently been revised by Efstathiou \& Siebenmorgen (2009)

\subsection{AGN torus models}

The most important constraint on early models for the torus in AGN (Pier \& Krolik 1992, Granato \& Danese 1994, Efstathiou \& Rowan-Robinson 1995) was provided by midinfrared spectroscopic observations from the ground in the $8-13 \mu \mathrm{m}$ window by Roche, Aitken and collaborators (Roche et al. 1991 and references therein). The observations showed moderate absorption features in type 2 AGN and featureless spectra in type 1 AGN. By comparison the flared discs (whose thickness increases linearly with distance from the central source) of Efstathiou \& Rowan-Robinson (1995)) and Granato \& Danese (1994) showed strong silicate emission features when observed face-on. Efstathiou \& Rowan-Robinson (1995)) proposed that tapered discs (whose thickness increases with distance from the central source in the inner part of the disc but tapers off to a constant value in the outer disc) with a density distribution that followed $r^{-1}$ could give flat spectra in the mid-infrared for an opening angle of around $45^{\circ}$ and an equatorial optical depth 
at $1000 \AA$ of 1000 . Models with a smaller opening angle predicted face-on spectra with shallow silicate absorption features whereas models with a larger opening angle showed emission features. Recent Spitzer observations showed emission features in quasars (e.g. Hao et al. 2005, Hao et al. 2007, Siebenmorgen et al. 2005, Spoon et al. 2007). The tapered discs of Efstathiou \& Rowan-Robinson (1995), in combination with the starburst models of Efstathiou, Rowan-Robinson \& Siebenmorgen (2000) have been very successful in fitting the spectral energy distributions of a number of AGN even in cases where midinfrared spectroscopy was available (Alexander et al. 1999, Ruiz et al. 2001, Farrah et al. 2003, Efstathiou \& Siebenmorgen 2005).

In this paper we use a grid of tapered disc models computed with the method of Efstathiou \& Rowan-Robinson (1995). In this grid of models we consider four discrete values for the equatorial $1000 \AA$ optical depth $(500,750,1000,1250)$, three values for the ratio of outer to inner disc radii $(20,60,100)$ and three values for the opening angle of the disc $\left(30^{\circ}, 45^{\circ}\right.$ and $\left.60^{\circ}\right)$. The spectra are computed for 37 or 74 orientations which are equally spaced in the range 0 to $\pi / 2$.

The grid of tapered disc models, as well as the grid of starburst models discussed above, are available from $\mathrm{AE}$ on request (a.efstathiou@euc.ac.cy).

\subsection{Comparison with the average spectrum of Seyfert $2 s$}

We first explore whether the combination of the tapered disc and starburst models described above can fit the average spectrum of Seyfert 2s presented by Hao et al. 2007 . The observed spectrum has been determined by first normalizing the spectra at $15 \mu \mathrm{m}$ and then averaging. The AGN models have also been averaged in an attempt to mimic the averaging of the observed spectra.

We find that the best fit to the Seyfert 2 average is provided by a model that assumes a torus opening angle of $45^{\circ}$, an equatorial $1000 \AA$ optical depth of 750 and a ratio of outer to inner torus radii of 20 . The best fit starburst model assumes an initial optical depth of the molecular clouds $\tau_{V}$ of 200 and a starburst age of $72 \mathrm{Myr}$.

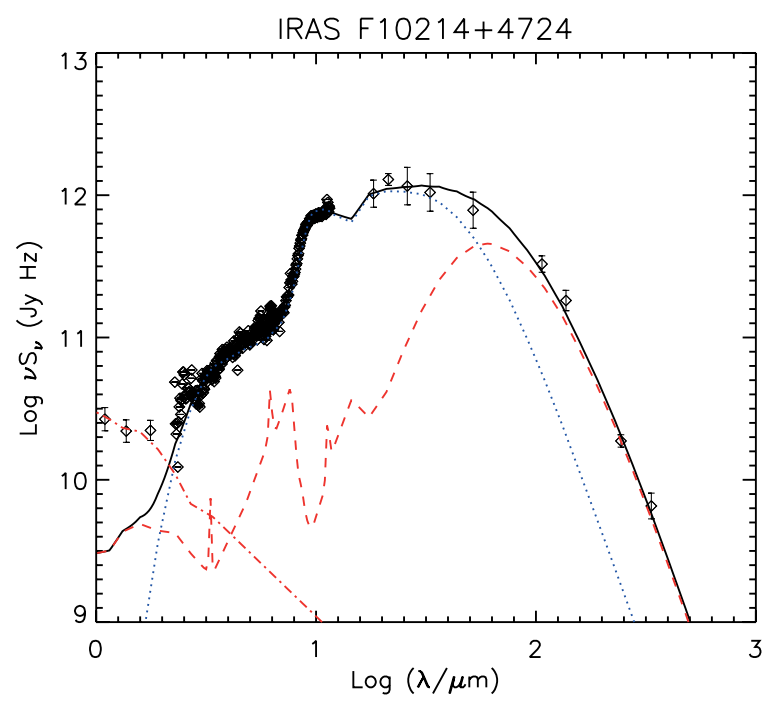

Figure 1. Fit to the spectral energy distribution of IRAS F10214+4724. The dotted and dashed lines show the contributions of the AGN and starburst respectively to the total emission (solid line). The dash dotted line also shows a fit to the IRAC data with a $1 \mathrm{Gyr}$ old stellar population. Data from Teplitz et al. (2006), IRAS, Rowan-Robinson et al. (1993), Sturm et al. (2010). 


\section{A new model for IRAS F10214+4724}

We find that the models described above provide a very poor fit to the SED of IRAS F10214+4724. This is clearly due to the fact that the SED of F10214+4724 shows simultaneously characteristics of almost edge-on and almost face-on emission. The failure of torus emission alone to fit the mid-infrared SED of F10214+4724 motivated Efstathiou (2006) to propose a three component model: an edge-on torus, clouds associated with the narrow-line region and a highly obscured starburst. The model assumed that the narrow line region clouds have a covering factor of $17 \%$ and two discrete values for the dust temperature (610 and 200K). The new MIPS and PACS data suggest that there is about a factor of two more power in the rest frame wavelength range 20-50 $\mu \mathrm{m}$ compared with the prediction of the model presented in Efstathiou (2006). Considering that the new data samples the peak of the SED of F10214+4724 this implies that the covering factor needs to increase even more. It therefore seems likely that the magnification of the emission from the narrow-line region is considerably higher than that from the torus. We find that the new data can be fitted by assuming an additional component of narrow-line region dust at a temperature of $80 \mathrm{~K}$. The new fit is shown in Figure 1. The torus emission is assumed to be negligible in this fit.

\section{References}

Alexander, D. M., Efstathiou, A., Hough, J. H. et al. 1999, MNRAS, 310, 78

Broadhurst, T. \& Lehar, J. 1995, ApJ (Letters), 450, L41

Efstathiou A. \& Rowan-Robinson M. 1995, MNRAS, 273, 649

Efstathiou A., Rowan-Robinson M. \& Siebenmorgen R. 2000, MNRAS, 313, 734

Efstathiou, A. \& Siebenmorgen, R. 2005, A\& A, 439, 85

Efstathiou, A. \& Siebenmorgen, R. 2009, A\&A, 502, 541

Efstathiou, A. 2006, MNRAS, 371, L70

Farrah D., Afonso J., Efstathiou A., et al. 2003, MNRAS, 343, 585

Genzel R. et al. 1998, ApJ, 498, 579

Granato G. L. \& Danese L. 1994, MNRAS, 268, 235

Hao, L. et al. 2005, ApJ (Letters), 625, L75

Hao, L. et al. 2007, ApJ (Letters), 655, L77

Pier E. A., \& Krolik J. H. 1992, ApJ, 401, 99

Roche P. F., Aitken D. K., Smith C. H., \& Ward M. J. 1991, MNRAS, 248, 606

Rowan-Robinson et al. 1991, Nature, 351, 719

Rowan-Robinson M. et al. 1993, MNRAS, 261, 513

Ruiz, M., Efstathiou, A., Alexander, D. M., \& Hough, J. 2001, MNRAS, 325, 995.

Siebenmorgen, R. \& Krügel, E. 1992, A\& A, 259, 614

Siebenmorgen, R., Haas, M., Kruegel, E., \& Schulz, B. 2005, A\&̈A, 436, L5.

Spoon, H. W. W., Marshall, J. A., Houck, J. R. et al. 2007, ApJ (Letters), 654, L49

Sturm et al. 2010, A\& A, 518, L36

Teplitz et al. 2006, ApJ (Letters), 638, L1

\section{Discussion}

TuFfs: AGNs are fairly randomly alligned with the dusty disks of their hosts. Do you expect measurable components of the FIR emission (for example at longer wavelengths) to be originating from diffuse dust in the disk illuminated by UV/optical from a favourable alligned AGN?

Efstathiou/Siebenmorgen: The heating of the diffuse dust of the host galaxies at kiloparsec scales by an AGN is discussed by Siebenmorgen et al. (2004); basically the 
luminosity of the AGN may be seen as a point source heating the dust with a flux scaling as $L_{A G N} / R^{2}$.

GROVES: If the IR NLR emission in F10214 is boosted by gravitational lensing wouldn't you expect the line emission to be relatively boosted as well?

Efstathiou: Lacy et al. (1998) also found that the emission lines in F10214 are particularly strong.

Thompson: A starburst of $\sim 100 \mathrm{M}_{\odot} / \mathrm{yr}$, on $\sim 100 \mathrm{pc}$ scales would produce $\sim 10^{13} \mathrm{~L}_{\odot}$, implying $\mathrm{T}_{\text {eff }} \sim 90 \mathrm{~K}$. Why couldn't such a starburst produce the $\sim 80 \mathrm{~K}$ component you require to explain the SED of IRAS F10214+4724?

Efstathiou: It would be very strange that we would then have two starbursts in this object. One with a temperature of $40 \mathrm{~K}$ and one with $80 \mathrm{~K}$. Such a starburst would also emit strong PAH features which would show up on the mid-infrared spectrum. 